ISSN: 2339-5753 | 2021 | Vol. 7 | pp. 2 I I-222

https://doi.org//0.5565/rev/aiet. 10 I

De la Gran Recesión a la Pandemia

\title{
Reseña de CASTILlo (DIR.) (2020). Cien AÑos del Ministerio de TRABAJO EN ESPAÑA
}

\section{Madrid: Ministerio de Trabajo y Economía Social, 388 páginas}

\section{Francisco Pérez Amorós}

Instituto de Estudios del Trabajo,

Universitat Autònoma de Barcelona, Spain

\section{Contacto}

Francesc.Perez.Amoros@uab.cat

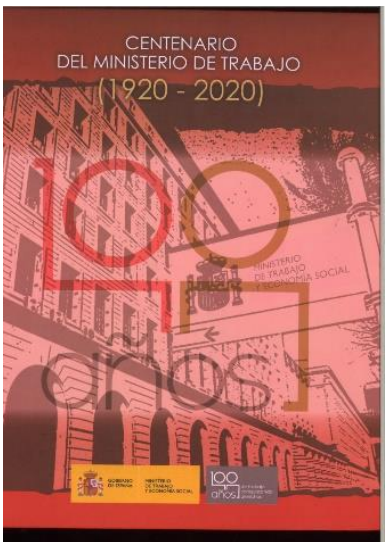

I. El libro, concebido como Catálogo de la Exposición del Centenario del Ministerio de Trabajo (1920-2020), contiene Presentación, Prólogo, diez ensayos, y una bibliografía final - con el soporte de fichas técnicas y fotografías-, que ofrecen informaciones y consideraciones sobre el Ministerio de Trabajo; y también es un soporte documental que - como un coffee table book, en el sentido de fuente de conocimiento de exquisito diseño- contextualiza y realza todos y cuantos actos se desarrollan con ocasión de dicha efeméride.

2. Estas páginas cumplimentan la cariñosa invitación que me formuló la profesora María Jesús Espuny Tomás, de la Universidad Autónoma de Barcelona, autora de uno de los capítulos de la obra. Deferencia que agradezco porque me permite seguir aprendiendo con su inestimable ayuda.

3. Esta recensión sintetiza los aspectos que cada autora o autor presenta como los más notables de la época estudiada, sin opinión alguna de quien la suscribe (o cuando menos así se procura), salvo las que se formulan en el epígrafe de cierre y la que añade a 
continuación. Se parte de la premisa - personal y expuesta en otros foros- que el Ministerio de Trabajo personifica en buena parte el "intervencionismo" (normativo, administrativo y judicial) que, junto con la "autonomía colectiva" y la "autonomía individual”, explica el porqué, el cómo y el para qué del Derecho del Trabajo, y define cualquier modelo de relaciones laborales.

4. En la Presentación - “Con la fuerza de cien años”-, la Ministra de Trabajo y Economía Social, Yolanda Díaz Pérez, recuerda que el Ministerio de Trabajo "es el que más reorganizaciones ha experimentado", e introduce la idea eje que los cien años de historia del Departamento transcurren con "tensiones dinámicas", la primera "la que enfrenta la realidad y su normativa, la ley y su aplicación”, y la segunda, "la que emana del diálogo social y la conflictividad laboral"; concluyendo la Ministra — con una sinécdoque - que "Trabajo (es) como (una) matriz argumental y reflejo de los cambios sociales y políticos [...] durante los últimos cien años" $y$, enlazando la historia con el futuro, plantea la necesidad de dotarse de un "Estatuto del Trabajo del siglo XXl", y reclama para su Departamento protagonismo para lograr avances sociales, contando, para ello, con "la vitalidad y con la fuerza de cien años".

5. El Prólogo "Cien años del Ministerio de Trabajo en España”, (sic cursivas en cabecera y línea final del Prólogo, pensamos que para enfatizar que se da cabida a todas las denominaciones que ha recibido), elaborado por el profesor Santiago Carrillo, presenta la sinopsis de cada una las diez grandes etapas — diez capítulos "con sólida base científica"de la vida del Ministerio de Trabajo, precisando, en cada una, los hechos que la identifican. Cada síntesis introduce la lectura del capítulo correspondiente, y un repaso de todas ofrece una visión general, útil a efectos varios. Apunta también el prologuista que el Ministerio en cuestión ha sido una institución "clave" para el "desarrollo de las políticas" y para la "regulación de las relaciones laborales", y que ha vivido "logros y/o fracasos".

6. En el primer capítulo, el Profesor Santiago Castillo trata de los "Antecedentes, creación y primeros pasos del Ministerio de Trabajo en España, 1920-1923”.

Resalta los "antecedentes" del Ministerio de Trabajo porque explican las causas de su "creación" (RD de 8 mayo 1920), que es tanto como decir que dejan ver sus funciones originarias: el "abstencionismo del estado", provoca un conflicto — la cuestión social-, que hace necesario el "intervencionismo del Estado", que el departamento ministerial de referencia pone en práctica, en parte.

Con "el arranque del intervencionismo [...] del Estado [...]" (intervencionismo científico) y el empuje del movimiento obrero, surgen unos primeros antecedentes. En el plano institucional, se crean, por ejemplo, el Instituto de Reformas Laborales (1903), el Servicio de Inspección de Trabajo (1906), el Instituto Nacional de Previsión (1908), y el Consejo Superior y la Inspección de Emigración (1907-1908). Y, “a partir de 1908” se produciría una primera "serie de reformas legislativas" (Leyes de Tribunales Industriales, 1908 y 1912 , 
Ley de Huelgas y Coligaciones, 1909; y Ley de Conciliación y Arbitraje Industrial, 1908), consideradas todas "piezas clave en la construcción de un nuevo sistema jurídico de relaciones laborales".

Con estos antecedentes, institucionales y legales, y en un "clima social ya hondamente crispado", se crea el Ministerio de Trabajo (RD de 8 mayo 1920), atendiendo así al llamado del Tratado de Versalles 1919 en favor de la administración laboral, y enlazando así su origen con el de la OIT. Se detalla en el estudio que tal Departamento pasaría después a denominarse Ministerio de Trabajo, Comercio e Industria (ex RD. 20 febrero 1922).

El funcionamiento del departamento ministerial durante sus tres primeros años fue apunta el Profesor Castillo- el propio de una "dificil infancia" resultado de "insuficiencias del sistema de relaciones laborales" que desembocaron en un "desbordamiento conflictivo", desarreglos todos que son el "telón de fondo de crisis global del sistema de la Restauración que propiciaría el establecimiento de la Dictadura militar de Primo de Rivera de 1923”.

7. El segundo capítulo, elaborado por la profesora María Jesús Espuny Tomás, versa sobre "El Ministerio de Trabajo durante la Dictadura de Primo de Rivera, 1923-1931: intervencionismo orgánico, legislación social y ordenación corporativa".

Durante el Directorio Militar (1923-1925), "Se desmantelaba el antiguo orden político" en general -el "Consejo de Ministros" se sustituía por subsecretarios-y el laboral en concreto, pues ocurría, por ejemplo, que ciertos asuntos del Ministerio de Trabajo, Comercio e Industria —así designado en la época - se debían someter "a la resolución del Presidente de Directorio como Jeje del Gobierno" (“dictadura unipersonal”). En el contexto de otras reformas administrativas, el Ministerio de referencia soporta prontamente sus primeras reorganizaciones internas (desarrollo de la administración laboral consultiva, en 1924, refundición en el Consejo de Trabajo del Instituto de Reformas Sociales, en 1924, y la propia reorganización del Ministerio de Trabajo, Comercio e Industria). Y el Departamento ministerial en cuestión auspicia, desde buen principio, la promulgación de normas novedosas ("la Ley y Reglamento de Emigración —Texto Refundido de 1924-", y el “Estatuto de Enseñanza Industrial”, 1924).

Durante el Directorio Civil (1925-193I) —expone la profesora Espuny- "Reaparece el Consejo de Ministros" (y se reinstauran los ministros). Se reorganiza la administración laboral y el - "todavía (de) breve vida"- Ministerio de Trabajo, Comercio e Industria, pasa a denominarse Ministerio de Trabajo y Previsión (1928). Así mismo, se promulgan novedades legislativas de relieve en variadísimas materias ("RDL de Trabajo a domicilio" de 1926 y el "Código de Trabajo" de 1926) y algunas otras "de carácter tutelar sobre seguro sociales". En esta línea de cambios se implementa la ordenación corporativa en el ámbito laboral ("Organización Corporativa Nacional" y "armonicismo social": Comisiones Mixtas y Comités Paritarios), que responde a "la intervención del Estado en el problema social [...] (y) la necesidad de estructurar el país en su aspecto económico". 
Se expone que, durante la Dictadura, España se incorpora a la Sociedad de Naciones, y firma ciertos convenios internacionales.

Al decir de la Profesora Espuny, son ocho años de historia — primorriverista- con un "Ministerio de Trabajo" que - con distintas denominaciones y hasta cinco veces reorganizado, tal y como se presenta en las fichas técnicas incluidas en el estudio- actúa a modo de "artífice de la política social de la Dictadura".

8. La profesora Josefina Cuesta cuida del tercer capítulo relativo a "El Ministerio de

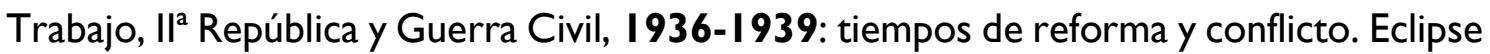
del ministerio en la España sublevada".

La II República — con su Constitución de 193I, ex ordinales I y 46- representa "un impulso sin precedentes a la política laboral...", es una etapa (193 I-1936) heterogénea con "continuos cambios [...] en el Ministerio de Trabajo, tanto en sus titulares, como en sus denominaciones, y en sus competencias", cambios pormenorizados en las fichas técnicas incluidas en el estudio.

El Ministerio de referencia, en el bienio I93 I-I933 ("Programa de la reforma social de $F$. Lago Caballero 193 I-1933”), protagoniza una "intensísima labor legislativa" sobre materias laborales trascendentales (jornada de 8 horas, 1931 y accidentes de trabajo, 1932), siendo "espectacular [...] el desarrollo de las relaciones colectivas" (Ley del Contrato de Trabajo de 21 de noviembre de 1931, Ley de Asociaciones profesionales de patronos y obreros de 1932, y la Ley de Jurados Mixtos de 1931), como intensa es también la legislación promulgada sobre la "unificación de los seguros sociales". Y, en el periodo 1934 a 1936 -bienio "rectificador" (“conservador”), según detalla la profesora Cuesta—, cambia de denominación y objetivos, procurando "la rectificación parcial de la legislación reformista del primer bienio", todo ello en un contexto de creciente de paro y de conflictos, algunos graves. En el estudio se detalla que España, durante este bienio - como en los tres años anteriores y como ocurrirá hasta 1939- seguía ratificando Convenios de la OIT, tal y como refleja en el estudio (cuadro o ficha número 4).

Durante la "Guerra Civil 1936-1939", y post Fuero del Trabajo de 9 de marzo de 1938 — “una obra principal"-, el Ministerio en cuestión emprende "una decidida política reformista"; y, durante un tiempo, "las competencias de Trabajo en la zona sublevada" (duplicidad de gobiernos), se atribuyen - "subsumidas" - al en el momento denominado "Ministerio de Organización y Acción Social" (ex Ley 30 enero 1938), resultando así —un "eclipse"- que "los órganos de Gobierno de la España sublevadas prescindieron de la cartera de Trabajo. Todo un símbolo de su ideario y preocupaciones". El estudio de la profesora Cuesta se cierra exponiendo que el Ministerio de Trabajo — tras su "eclipse"- "se restablecía" como tal (Ley 8 agosto 1939) debidamente reorganizado (Decreto 18 agosto 1939).

9. Bajo el título "Ministerio de Trabajo, 1939-1957: de la trinchera al franquismo", el profesor Pedro González Murillo presenta el cuarto capítulo del libro. 
La política social de la época tiene como "punto de partida" el Fuero del Trabajo (1938), y sus cimientos en el Ministerio de Trabajo, restablecido y reordenado en 1939 (Ley 8 agosto y Decreto de 18 agosto, respectivamente), que intentaba separar el "mundo del trabajo del estrictamente sindical" (supresión del Ministerio de Organización y Acción Sindical, pasando a Trabajo algunas de sus competencias).

El Ministerio en cuestión seguía desplegando su protagonismo, realzado en ocasiones por las tensiones derivadas de las interferencias entre sus funciones y las ("sindicales" y "falangistas") de la denominada "Organización Sindical Española", que en la época (en la que "el movimiento sindical obrero tradicional no tenía cabida") era un sindicato que funcionaba bajo los principios de "unidad, totalidad y jerarquía" (Ley de Unidad Sindical de 1940 y Ley Bases la Organización Nacional Sindicalista, 1940).

Se aprueban normas laborales relevantes: la "Ley de Reglamentaciones Laborales de 1942" que reguló el modelo de la "las relaciones laborales [...] hasta su quiebra con la promulgación de la Ley de Convenios Colectivos de 1958”, dando principio así al final de un ciclo; la "Ley de Contrato de Trabajo de 1944"; la represión —-penal— de la huelga ("Código Penal 1944"); la norma de creación de los "Jurados de Empresa" (en 1947, reglamentados en 1953, e implementados en 1957) "intentando armonizar el mundo del trabajo"; y la puesta en práctica de la "Magistratura de Trabajo" (ex Decreto I 3 mayo 1938), "instancia [en la que] se enquistaron lacras que imposibilitaron la defensa real [...] de los trabajadores". El repetido Ministerio de Trabajo también se identifica por auspiciar normas con otro tipo de contenido distinto: sobre la promoción de la "vivienda para los trabajadores"; la "educación a los obreros"; y la "política de previsión social" con "base de un conjunto descoordinado de seguros asentados en un criterio profesional, no universal"). Como argumenta el autor del capítulo, se trata de la legislación de una dictadura (con cierto "rostro social": "que compaginó represión y control"), que configura la "política reglamentista e intervencionista del franquismo en materia de trabajo".

El profesor González Murillo, en la Introducción de su estudio, ya anticipaba que "El régimen de 18 de julio [...] [de la] mano de la represión [...] [intenta] atraer a las masas obreras al redil del franquismo", a cuyo fin el "Ministerio de Trabajo" pone en práctica una política que hace del "trabajo y su entorno [...] un factor de armonización social con la intervención del Estado". Un planteamiento que se caracterizó "tanto por sus realizaciones tangibles e innegables como por la distancia sistemática — también tangible e innegableentre el relato construido por el régimen y los resultados efectivos de sus políticas".

10. El quinto capítulo, intitulado "El Ministerio de Trabajo, 1958-1976. Etapa de transformaciones: del desarrollismo económico franquista al inicio de la transición”, está redactado por el profesor Jorge Torrents Margalef.

Es una etapa limitada por dos leyes (Ley Convenios Colectivos, 1958 y Ley de Relaciones Laborales, 1976), que se corresponde a "un segundo gran tramo de la dictadura", y que finaliza a la par que el franquismo "de la mano de la [...] Ley para la Reforma Política" (4 de enero 1977). Es una época marcada por sus contradicciones internas tanto políticas 
(Ley Orgánica a del Estado de 1967 versus aperturismo) como económicas ("autarquía" versus "desarrollismo económico"), que repercuten en la legislación política y "[condicionan] las medidas del Ministerio de Trabajo" que actúa muy constreñido por una organización sindical que actúa bajo la autoridad de un Ministerio de Relaciones Sindicales (I97I 1977) cuyas "funciones sindicales (son) próximas al Ministerio de Trabajo" (ex Ley Sindical de 197I).

El Profesor Torrents valora las principales actuaciones del Ministerio de Trabajo: la implementación del "salario mínimo interprofesional"; la reducción del "poder normativo del Ministerio de Trabajo" (Reglamentaciones) debido a "la instauración de la negociación colectiva" (Ley de 1958 citada)"; la "permisión de los conflictos colectivos", y la celebración de "elecciones sindicales" (Jurados de Empresa), que facilitan "el entrismo" y dan auge a un "modelo de organización obrera, democrática y de clase" (tolerancia y permisión); y la organización de "el paso de la Previsión Social a la Seguridad Social" (normas básicas dictadas al efecto desde 196I a 1966).

Llegados aquí, el estudio concluye con la aprobación de la Ley de Relaciones Laborales de 18 de abril de 1976, a la que siguen las normas que dan lugar, en expresión del profesor Torrents, al "desmantelamiento institucional" del Ministerio de Trabajo de la época, un final ministerial que coincide en el tiempo - en el estudio- con la aprobación de la Ley para la Reforma Política (4 enero 1977) que conduce "Hacia el final del franquismo" $y$ al inicio de la democracia.

I I. El profesor Fernando Valdés Dal-Ré dedica el sexto capítulo al estudio de la "Historia del Ministerio de Trabajo, 1977-198I. El comienzo y la consolidación de la Democracia”.

El autor, asciende a la categoría de rúbrica, la ecuación que une, estrechamente, la figura del Ministerio de referencia a la Democracia.

Durante este periodo - “el final de la transición política": primeras elecciones democráticas, 1997 y promulgación de la CE, 1978-, el Ministerio de Trabajo (1977 a 198I), pasó a ser, tras varias reformas, Ministerio de Trabajo y de Sanidad y Seguridad Social (198I de marzo a noviembre) y, finalmente, Ministerio de Trabajo y Seguridad Social (noviembre-diciembre 1981, hasta 1996).

El Departamento ministerial referido resulta ser: "un agente político de primer orden”; un actor que alcanza y ejerce un "notorio alcance orgánico y funcional" (con citados cambios de denominación y reorganizaciones internas profundas); y una fuente con un "hiperactivismo normativo" de "notable trascendencia” laboral (RDLRT, 1977 y LET, 1980), en materia de empleo (LBE, 1980), de seguridad social (RDL 36/I978), y, procesal laboral (LPL, 1978).

Según el estudio del profesor Valdés, el Ministerio de referencia despacha tres "asuntos" que "desempeñaron, e incluso siguen desempeñando, una posición de máxima centralidad en el sistema español de relaciones laborales”. Promociona la Ley 19/1977, de 30 de marzo, 
regulando el derecho de Asociación Sindical ("reforma sindical"): "una pieza imprescindible del cambio político", que es "el punto final al proceso de liquidación de las estructuras [...] del sindicalismo vertical, eliminado la figura del Ministerio de Relaciones Sindicales y atribuyendo sus funciones y competencias el Ministerio de Trabajo", que refuerza su renovado protagonismo con el dictado de normas básicas (RDLRT, 4 de marzo de 1977) y la ratificación de fuentes internacionales (C 87 y 98 OIT, por ejemplo). Así mismo, dicho Ministerio participa del reconocimiento "del derecho de huelga" (RDLRT de 1977 y STC I I/। 98 I, 8 abril). Y, en tercer lugar, el autor destaca el protagonismo ministerial en la aprobación -y constante reforma- de la Ley 8/1980, de 10 de marzo, del Estatuto de los Trabajadores, de cuya "centralidad política y jurídica" en el "entramado normativo" y en "el propio modelo de relaciones (laborales)" se da cuenta en el estudio, una norma que en principio generó una "confrontación política y disenso sindical" y que al final mereció "La aprobación, por amplio consenso".

Se subraya en el estudio, que "el diálogo social y consenso político" se refleja en el Acuerdo Básico Interprofesional (UGT Y CEOE, julio 1979), que sería "el primer acuerdo interprofesional del período constitucional".

Nos dirá el profesor Valdés que, por su relación con la democracia y su prolífica actividad, el "periodo 1977-198I constituye uno de los más importantes en la historia del Ministerio de Trabajo".

12. El profesor Manuel Carlos Palomeque López, aporta el capítulo séptimo centrado en "Los primeros gobiernos socialistas de la Democracia, 1982-1996".

Tras "las elecciones legislativas [...] del 28 de octubre de 1982" ("primer gobierno socialista": "la Transición democrático había sido completada..."), se inicia "una larga etapa de reformas políticas y sociales", de "la economía, el empleo y las relaciones laborales" que concluirá con las "elecciones generales de marzo de 1996" (el PP obtiene mayoría simple). En el marco de la reforma de la administración pública de 1982 (RDL 22/I982, de 7 diciembre), el Ministerio de Trabajo y Seguridad Social readecuada su estructura y renovados sus objetivos, se convierte en una pieza de referencia de la misma.

La etapa coincide con el "ciclo reformador de adaptabilidad normativa" abierto por la "reforma laboral de 1984" (Ley 32/I 984 de 2 de agosto) que posibilitaba la "flexibilización del marco regulador del mercado de trabajo", y que continuaría con otra "gran" reforma laboral en 1993-1994 (Ley I I/1994, 19 mayo) favorecedora de la "adaptabilidad [...] de las relaciones de trabajo [...] [para] mejorar su competitividad y facilitar el empleo".

Durante el periodo se aprueba la Ley Orgánica de libertad sindical (LO I I/I985, 2 agosto), siendo la cuestión de la "representatividad sindical", en sus distintas variantes, una de las cuestiones controvertidas de tal norma ante el Tribunal Constitucional. La etapa considerada, según expone el profesor Palomeque, también se caracteriza por la "incorporación de España a las Comunidades Europeas" (LO I0/I985/ de 2 de agosto), que establece condicionantes del "Derecho de la Comunidad Europea sobre el ordenamiento interno"; 
y por la preparación de normas "de transferencia de servicios y medios" en materia laboral a las Comunidades Autónomas.

Son tiempos de controversias laborales, incluido el "enfrentamiento" "sindical" con "el Partido Socialista, ahora Gobierno" y la "huelga general 1988"; pero también lo son de "diálogo y concertación social" que dan lugar a "grandes pactos nacionales" (AI, I983; AES, I984) y a la "concertación bilateral", y a otra concertación más institucionalizadas ("creación del CES, 199I"). Sigue el estudio recordando que, fracasados los intentos "sobre la promulgación de una nueva ley huelga y de medidas de conflicto colectivo" (1992-1993)", la huelga seguirá regulada por una norma preconstitucional (RDL 17/1977) y depurada por el TC (198I); y se refiere también al empeño ministerial por la "culminación del proceso de sustitución de las reglamentaciones y ordenanzas laborales franquistas" (Ley I I/I994) por convenios colectivos, proceso que el Profesor Palomeque tilda de lento e incompleto.

13. "El Ministerio de Trabajo y Asuntos Sociales, 1996- 2004: Política social y reforma laboral en los gobiernos Aznar”, es el título, del octavo capítulo presentado por el profesor Joaquín García Murcia.

El Departamento en cuestión mantiene su protagonismo: sufre una "reorganización interna" (la Seguridad Social se asigna a una Secretaría de Estado: "no se erigió en un Ministerio"), pasa a ser "Ministerio de Trabajo y Asuntos Sociales", dando lugar así a un "nuevo Ministerio", que será objeto de "reestructuraciones" profundas varias (1996, 1998 y 2000), aunque retendrá la misma designación durante toda la etapa.

El autor señala que "Uno de los más importantes compromisos del nuevo Ministerio [...] fue [la] [...] concertación [...] con las organizaciones sindicales y empresariales": dichos débitos —adquiridos por "convicción" y por "conveniencia política"-, se cumplimentan mediante la "concertación social en sentido estricto" y la "concertación social bilateral" que dan lugar acuerdos varios (Pactos Moncloa, 1997; AES, 1983; y, ANC, 1997 y 2002).

A la par, el Ministerio referido despliega una "intervención normativa" abundante en materia laboral ("mejora" y "reordenación del mercado de trabajo: reforma laboral”, 1997 y 200 I, y Ley Empleo 16 diciembre 2003); en cuestiones de seguridad social en general (a partir de la Ley 24/I997, 15 junio, para la consolidación y racionalización del sistema); y, en particular, para la "protección por desempleo", y la implantación de la "renta activa de inserción". Así mismo, varios son los "instrumentos normativos" dictados sobre materias más concretas ("conciliación, igualdad de trato y tutela de discapacitados"; "ordenadora de la Inspección de Trabajo y Seguridad Social, 1997"; y reguladora de "Infracciones y Sanciones del Orden Social, 1982”).

En el "Epílogo”, con el que el que el profesor García Murcia cierra el capítulo, se subrayan dos trazos característicos de la época considerada: "que la producción normativa en materia de empleo, relaciones de trabajo y seguridad social alcanzó niveles considerablemente altos"; y que se hacía "uso relativamente intenso de la legislación de urgencia para efectuar reformas en el ámbito de la legislación laboral”. Y, haciendo "la valoración (del) Ministerio de 
Trabajo", asevera el autor "que siempre ha sido puntero", "lo mismo que no cabe poner reparos al nivel de diligencia mostrado particularmente por el Ministerio de Trabajo y Asuntos Sociales durante aquel periodo", precisando que "Naturalmente, puede debatirse mucho acerca de la orientación ideológica, de los principios de política económica o de las opciones de gasto social" de la política de los gobiernos de la época considerada.

I4. "El Ministerio de Trabajo, 2004-20I I: de la esperanza del pleno empleo a la contención de los efectos devastadores de la crisis económica", es el noveno capítulo del libro, y ha sido elaborado por la profesora María Luz Rodríguez Fernández, que, se debe recordar, fue Secretaria de Estado de Empleo del 30 de octubre de 2010 al 30 diciembre de 2011 .

Durante el periodo 2004-2008 ("bonanza económica y “crecimiento del empleo"), el citado Ministerio de Trabajo y Asuntos Sociales, como "protagonista de la conquista de los nuevos derechos sociales", centra su actividad, según expone la profesora Rodríguez Fernández, en: las "políticas de igualdad", ("Ley Orgánica I/2004, de 28 diciembre, de Medidas de Protección Integral contra la Violencia de Género”, y Ley Orgánica 3/2007, de 22 de marzo, para la igualdad efectiva de mujeres y hombres); la "promoción y atención a las personas en situación, de dependencia" (Ley 39/2006, I 4 diciembre, sobre "personas en situación de dependencia": un "intento de construir el cuarto pilar del Estado Social", que "apenas tuvo realización práctica"); y en las políticas de empleo ("mejora del crecimiento y del empleo": "temporalidad" versus "estabilidad"). En el estudio también se significa la "expansión de los derechos laborales a autónomos" (“Ley 20/2007, I I de julio, del Estatuto del trabajador autónomo"), y a "personas inmigrantes indocumentadas" (RD 2393/2004, de 30 de diciembre que aprueba el Reglamento de la Ley Orgánica 4/2000 de "extranjería”); y, se comenta el "Acuerdo para la mejora del crecimiento y del empleo" ( 9 mayo de 2006).

Durante 2008-20I I ("crisis económica"), el ahora denominado "Ministerio de Trabajo e Inmigración", que pierde ciertas competencias, pero refuerza las suyas sobre políticas activas de empleo, e inmigración (LO 2/2009, I I de diciembre, de reforma de la Ley de 2000 de "extranjería"), se sitúa en "en el epicentro de las políticas para hacer frente [...] [a] la crisis económica", y da paso a la "reforma laboral de 2010" (RDL 10/2010, 16 de junio, transformado en la Ley 35/2010, 17 de septiembre), implementada sin "acuerdo alguno en el diálogo social" (huelga general del 29 de septiembre 2010). Y sigue la autora exponiendo que, tras los cambios producidos en octubre 2010 en el referido Ministerio, se intenta "recuperar el diálogo social con los actores sociales" (AES, de 2 de febrero 20II), y se proponen "tres reformas fundamentales" ("pensiones, [...] la negociación colectiva y [...] las políticas activas de empleo"), con prácticas y resultados desiguales debido a la crisis económica (RDL 20/20I2, I3 julio, medidas para garantizar estabilidad presupuestaria y fomento competitividad).

La profesora Rodríguez Fernández ya había anunciado desde buen principio que "El Ministerio tendrá un papel central en la vida política", como "protagonista" del establecimiento 
de nuevos derechos o como actor situado en "epicentro" frente a la crisis, según las circunstancias.

I5. El último capítulo del libro - “El Ministerio de Empleo y Seguridad Social, 20 II2018. Gestión de la crisis, empleabilidad y Futuro del Trabajo"- es obra de la profesora Nuria P. García Piñero.

Los primeros años (20II-20I5) de la etapa - “Crisis económica y proceso de reformas", son también, de "elevado paro". El precitado Ministerio — con una denominación "distinta al tradicional Ministerio de Trabajo"-, "afronta en un momento delicado [...] una importante tarea en todos los ámbitos" (empleo, Seguridad Social... extranjería, inmigración y emigración). La firma del II Acuerdo para el Empleo y la Negociación Colectiva de 25 enero 2012, ante un desempleo muy elevado, "no logró evitar la reforma laboral" de 2012 (RDL 3/20I2, 10 de febrero) que, inspirada en la "flexibilidad" y la "flexiseguridad", tiene —según la autora - cuatro objetivos, ("empleabilidad", "flexibilidad interna", "contratación indefinida", y a "eficiencia del mercado y reducir la dualidad"), y cuya aprobación "llevó a dos huelgas generales" (29M-20I 2 y I4N-20I2). La autora plantea que la "flexiseguridad compensa ciertas rebajas flexibilizadoras de los derechos laborales"; apunta que "el diagnóstico y la reforma laboral del Partido Popular son muy similares a los de las dos últimas reformas del Partido Socialista de 2010-20I I"; y sostiene que "sin crecimiento económico es muy dificil crear puestos de trabajo, limitándose la normativa laboral a coadyuvar en la consecución del tal objetivo".

El trabajo de la profesora García Piñero estudia las novedades normativas patrocinadas por el Ministerio de referencia durante los años 20I I-20I5: "contratación” laboral; "empleo juvenil" (Estrategia Empleo Joven); "autoempleo y emprendimiento"; "lucha contra el fraude y trabajo irregular"; "protección por desempleo asistencial"; "políticas activas de empleo"; "formación profesional para el empleo"; "sostenibilidad del sistema de la Seguridad Social"; y en materia de "Inmigración y emigración". En 20I4, "la economía volvió a crecer y se volvió a crear empleo" y el paro desciende, en un año, "más de dos puntos porcentuales" y se sitúa en $23,70 \%$ en el 4 T 20I4, según consta en el estudio. El 2015 es el año de "la firma del III Acuerdo para el Empleo y la Negociación Colectiva 20 I 5-20 I 7", y de "la racionalización, simplificación y modernización de la legislación laboral y de la seguridad social" ("Textos refundidos de la LET, de la Ley de Empleo, y de la Ley de la Seguridad Social”).

En un segundo período (enero a julio de 2016: “Crisis política y Gobierno en funciones”), el Ministerio en cuestión, principalmente, "se limitó al despacho ordinario de los asuntos [...] absteniéndose de adoptar cualesquiera otras medidas". Y, en un tercer periodo (2016-2018: "recuperación económica y del empleo") — según se expone en el estudio, bajo el epígrafe "El Futuro del Trabajo—, "el Ministerio vuelca su preocupación [...] nuevamente por el desempleo juvenil" ("Sistema Nacional de Garantía Juvenil”), aprueba medidas para favorecer el "trabajo autónomo" y "la conciliación de la vida laboral y familiar", y adopta "medidas relativas a formación profesional para el empleo", previsiones estas truncadas, en buena 
parte, por la finalización imprevista de la legislatura (moción de censura al Gobierno de 4 junio 20I8).

La profesora García Piñero, afirma que "La importancia del Ministerio de Empleo y Seguridad Social destaca en el conjunto del Gobierno, tanto cuantitativamente como cualitativamente".

16. En opinión de quien suscribe, el libro "Cien años del Ministerio de Trabajo (19202020)", suscitará muchas y variadas sugerencias a sus lectores, algunas de presente y otras de futuro y, en su caso - seguro-, también motivará vivos recuerdos del pasado: la historia, como se ha escrito, tiene eso, une el pasado, el presente y el futuro.

El libro presenta a su homenajeado - el Ministerio de Trabajo- como uno de los protagonistas de las distintitas etapas de la historia de tal centuria mostrando así otra faceta de la centralidad del factor trabajo. Recuerda el libro que el sentido protector del primer ordenamiento laboral ya se debía, en parte, a la actividad de la Administración laboral (Alonso García, 1957) y que así siguió ocurriendo en casi todas las épocas posteriores (Mercader Uguina y Tolosa Tribiño, 2004), y da a entender que, hoy día, el intervencionismo del Ministerio de Trabajo previsto constitucionalmente (ex artículo 9.2 de la Constitución, Rodríguez-Piñero y Bravo-Ferrer, 2009) e internacionalmente (C I50/1978 OIT, Vida Soria, 2009), sigue operativo en favor de la función tuitiva de las normas e instituciones laborales (Babiano Mora y Tébar Hurtado, 2020). Y el libro sugiere que el listado completo de todas las denominaciones recibidas por el Ministerio de Trabajo a lo largo de sus - casi - cien años de vida (incluida la denominación que no lo incluye literalmente en una clara "huida del trabajo"), conforman un vademécum sobre su historia; a la vez que el libro conmemorativo abona la idea que todas dichas denominaciones, se encierran en una: "Ministerio de Trabajo", pero de un "Trabajo" que debería ser —realmente - "decente" (llámese como se llame), dicho sea trayendo a colación la también centenaria OIT (y recordando "la importancia de llamarse Ernesto").

El director del libro "Cien años del Ministerio de Trabajo (1920-2020)", como ha quedado dicho, subraya — subrayamos - que es un estudio de historia con una sólida base científica y, a buen seguro, se podría convenir que es un libro capital con valor futuro. El (primer) centenario del Ministerio de Trabajo: una historia con futuro.

\section{FRANCISCO PÉREZ AMORÓS}

Instituto de Estudios del Trabajo, Universidad Autónoma de Barcelona 
Referencia normalizada

Pérez Amorós, Francisco (2021). Reseña de Castillo (dir.) (2020). Cien años del Ministerio de Trabajo en España. Anuario IET de Trabajo y Relaciones Laborales, 7, 21 I-222.

https://doi.org/I0.5565/rev/aiet. I0I 